\title{
Studies of Unstable Nuclei with Spin-Polarized Proton Target
}

\author{
Satoshi Sakaguchi ${ }^{1,2, *}$, Tomohiro Uesaka ${ }^{2}$, Takashi Wakui ${ }^{3}$, Sergey Chebotaryov ${ }^{2,4}$, Tomomi \\ Kawahara $^{2}$, Shoichiro Kawase ${ }^{5}$, Evgeniy Milman ${ }^{2,4}$, Tsz Leung Tang ${ }^{5}$, Kenichiro Tateishi ${ }^{2}$ \\ and Takashi Teranishi ${ }^{1}$ \\ ${ }^{1}$ Department of Physics, Kyushu University, 6-10-1 Hakozaki, Higashi-ku, Fukuoka-shi, \\ Fukuoka 812-0053, Japan \\ ${ }^{2}$ RIKEN Nishina Center, 2-1 Hirosawa, Wako-shi, Saitama 351-0198, Japan \\ ${ }^{3}$ Cyclotron and Radioisotope Center, Tohoku University, 36-3 Aoba, Aramaki, Aoba, Sendai, \\ Miyagi 980-8578, Japan \\ ${ }^{4}$ Department of Physics, Kyungpook National University, 80 Daehakro, Bukgu, \\ Daegu, 702-701, Korea \\ ${ }^{5}$ Center for Nuclear Study, University of Tokyo, 7-3-1 Hongo, Bunkyo-ku, \\ Tokyo 113-0033, Japan \\ *sakaguchi@phys.kyushu-u.ac.jp
}

Published 29 February 2016

\begin{abstract}
Roles of spin-dependent interactions in unstable nuclei have been investigated via the direct reaction of radioactive ions with a solid spin-polarized proton target. The target has a unique advantage of a high polarization of $20-30 \%$ under low magnetic field of $0.1 \mathrm{~T}$ and at a high temperature of $100 \mathrm{~K}$, which allow us to detect recoil protons with good angular resolution. Present status of on-going experimental studies at intermediate energies, such as proton elastic scattering and $(p, 2 p)$ knockout reaction, and new physics opportunities expected with low-energy RI beams are overviewed.
\end{abstract}

Keywords: Polarized target; unstable nuclei; direct reaction.

PACS numbers: 25.60.Bx, 24.70.+s, 25.40.Cm, 29.25.Pj, 21.10.Pc

\section{Introduction}

The world of atomic nuclei is enriched by the strong spin-dependent interaction in the nuclear force. Spin-orbit, tensor and three-body forces play essentially important roles in nuclear structure and reaction. For studying such interactions, one of the powerful approaches is the direct reaction of spin-polarized light nuclei. From more than half century ago, a number of scattering experiments using polarized proton/deuteron beams have been performed in all over the world. On the other hand, study of nuclei far from the stability line has been extensively carried out for these

This is an Open Access article published by World Scientific Publishing Company. It is distributed under the terms of the Creative Commons Attribution 3.0 (CC-BY) License. Further distribution of this work is permitted, provided the original work is properly cited. 


\section{S. Sakaguchi et al.}

three decades almost independently with such spin physics. Various exotic phenomena and property of nuclei have been discovered: for example, appearance and disappearance of magic numbers. The spin-dependent interactions attracts renewed interest as they are pointed out to be key elements underlying these phenomena. ${ }^{1-4}$ At RIKEN and CNS University of Tokyo, we have constructed a solid spin-polarized proton target that is applicable to RI-beam experiments, ${ }^{5}$ which allows measurement of polarization observable in the direct reaction involving unstable nuclei. This article describes present status of the studies of unstable nuclei with the polarized target such as determination of spin-orbit potential by proton elastic scattering and investigation of spin-orbit splitting by $(p, 2 p)$ knockout reaction. New physics opportunities expected in low-energy RI-beam experiments is also covered.

\section{Solid Polarized Proton Target for RI-Beam Experiment}

\subsection{Requirements for target}

The polarized target for RI-beam experiments has to be operated under a low magnetic field of about $0.1 \mathrm{~T}$ so that low-energy recoil particles can be detected with good angular resolutions under the inverse-kinematics condition. On the other hand, conventional polarized proton targets utilizing electron polarization caused by Boltzmann distribution method are operated under much higher magnetic field of about $2.5 \mathrm{~T}{ }^{6}$ While one of the approaches to achieve a high proton polarization under low magnetic field would be a frozen-spin-target technique, it requires a very low temperature of about $100 \mathrm{mK}$, which is not easy to keep during the heavy-ion beam irradiation.

\subsection{Triplet-DNP method for high spin-polarization under low magnetic field}

In order to realize a solid spin-polarized proton target under low magnetic field, we adopted a dynamic-nuclear-polarization technique making use of photo-excited triplet states of pentacene (Triplet-DNP $\operatorname{method}^{7}$ ). In this method, spin polarization of electrons occurs spontaneously between Zeeman sublevels of the triplet states. Since the value of the polarization is almost independent of the magnetic field strength and temperature, high electron-spin polarization of $70-90 \%^{8}$ can be attained even in a relaxed conditions: a low magnetic field of $0.1 \mathrm{~T}$ and at high temperature of $100 \mathrm{~K}$. The electron-spin polarization is transferred to the proton system by a cross-relaxation technique. ${ }^{9}$

Polarized target based on the triplet-DNP method has been first realized at Kyoto University, ${ }^{10}$ and then adopted for the polarized target for RI-beam experiments at RIKEN and CNS, Univ. of Tokyo. ${ }^{5}$ Recently, triplet-DNP method has been applied also to a broadband neutron spin filter at Paul Scherrer Institute. ${ }^{11}$ 


\subsection{Solid spin-polarized proton target at RIKEN}

The solid spin-polarized proton target constructed at RIKEN and CNS, Univ. of Tokyo is overviewed. The target material is a disk-shaped single crystal of naphthalene. Diameter and thickness of the target are 14-24 $\mathrm{mm}$ and 1-3 $\mathrm{mm}$, respectively. Large diameter is required for minimizing background events from the target holder and also to maximize the statistics. Pentacene molecules are doped in the crystal with a concentration of $\sim 0.005 \mathrm{~mol} \%$ as sources of electron-spin polarization. To suppress the relaxation rate of the proton-spin polarization, the crystal is cooled down to $100 \mathrm{~K}$ in a double-folded chamber by the flow of cold nitrogen gas. The chamber is placed at the center of a C-type electromagnet, which supplies a static magnetic field of 60-120 mT to define the polarization axis. Electron-spin polarization is produced by an unpolarized pulsed laser light with a wavelength of $514 \mathrm{~nm}$, and transferred to the proton system by microwave irradiation. Thin loop-gap resonator made of $25-75 \mu \mathrm{m}^{t}$ teflon and $4.4-36 \mu \mathrm{m}^{t}$ copper is used as a microwave resonator so that the recoil proton can penetrate it. The magnitude of proton-spin polarization is monitored by a pulse-NMR method and calibrated using measured spin asymmetry of $\vec{p}+{ }^{4} \mathrm{He}$ or $\vec{p}+p$ scattering. Typical polarization during the beam time is $20-30 \%$, which corresponds to the enhancement of five-orders-of magnitude relative to the polarization under the thermal equilibrium.

\section{Studies of Unstable Nuclei at Intermediate Energy}

The solid spin-polarized target has been applied to several RI-beam experiments at intermediate energies of 70-250 MeV/A at RIKEN RI Beam Factory. Vector analyzing powers have been measured for the proton elastic scattering and for the $(p, p N)$ nucleon knockout reaction to investigate the nature of spin-orbit coupling in the reaction and structure of unstable nuclei.

\subsection{Proton elastic scattering}

One of the direct manifestation of the spin-orbit coupling in atomic nuclei is the polarization phenomenon in the nucleon elastic scattering. It causes the asymmetry of the cross section depending on the direction of nucleon spin as $A_{y}=\frac{1}{P_{y}} \frac{\sigma_{\uparrow}-\sigma_{\downarrow}}{\sigma_{\uparrow}+\sigma_{\downarrow}}$, where $A_{y}, P_{y}$, and $\sigma_{\uparrow / \downarrow}$ are the vector analyzing power, polarization of probe, and spin-up/down cross sections, respectively. The analyzing power of proton elastic scattering on stable nuclei have been widely measured using the double scattering method first and then using the polarized proton beam. Spin-orbit coupling in the elastic scattering is well established at present in the region of stable nuclei. The spin-orbit part of the optical potential is modeled by the radial derivative of the nucleon density distribution and known to have a peak at the nuclear surface with a depth of $\sim 5 \mathrm{MeV}$. It would be interesting to study how the spin-orbit potential changes in very neutron-rich nuclei that have diffuse density distributions.

Analyzing power measurement for the $\vec{p}+{ }^{6} \mathrm{He}$ and ${ }^{8} \mathrm{He}$ elastic scattering have been carried out at $71 \mathrm{MeV} / \mathrm{A}$ at RIPS in RIKEN. ${ }^{12-14}$ These isotopes are known 


\section{S. Sakaguchi et al.}

to have a neutron-halo/skin structure. The optical model analysis revealed that the spin-orbit potentials between a proton and neutron-rich helium isotopes have shallow and extended radial dependence compared with the global systematics of stable nuclei. This feature can be understood from the diffuse density distribution of these nuclei and from the center-of-mass motion of the $\alpha$ core. ${ }^{12,13}$

While the gross characteristics of the spin-orbit potential has been obtained in the phenomenological analysis, it is important to establish understanding of the elastic scattering based on sophisticated reaction models. Two folding model calculations have been performed at $71 \mathrm{MeV} / \mathrm{A}$ using $t$ - and $g$-matrices as an effective interaction. The latter gives better reproduction of the analyzing power data probably because of the proper treatment of the non-locality of the exchange potential. This implies the complexity of the reaction mechanism in this energy region and that the data at higher energy would be valuable for clearer understanding of the relevance of spin-orbit coupling with the nucleon density distribution. We plan to measure the $\vec{p}+{ }^{6} \mathrm{He}$ analyzing power at higher energy of $200 \mathrm{MeV} / \mathrm{A}$ at RIKEN RI Beam Factory. SAMURAI spectrometer will be used for the clearer identification and for momentum analysis of the scattered particles. Target diameter is planned to be enlarged from $14 \mathrm{~mm}$ to $24 \mathrm{~mm}$ to improve the signal-to-noise ratio. Microscopic understanding of the optical potential and the mechanism of elastic scattering will serve also as a basis for the analysis of other reactions such as inelastic scattering and knockout reaction.

\section{2. $(\vec{p}, p N)$ knockout reaction}

Spin-orbit coupling has been an essential ingredient also in the nuclear structure since the shell structure was introduced by Mayer and Jensen. Recently, there has been a renewed interest in it because the spin-orbit splitting is pointed out to be a key for the change of shell structure in unstable nuclei. It should be important to experimentally determine the spin-orbit splitting and investigate its systematics along the isotope chain to shed a light on the physics underlying this phenomenon.

Direct reactions such as transfer and knockout reactions have been powerful tools to study the distribution of single-particle strength. Here, polarization observables are particularly important because of their sensitivity to the total angular momentum $J$. For example, the vector analyzing power of the $(\vec{p}, p N)$ reaction changes its sign depending on the states with the spin parallel or antiparallel to the angular momentum because of the large spin-correlation parameter and of the absorption of a recoiled nucleon. ${ }^{15}$ The capability to determine $J$ is crucially important because one needs to take a weighted average of the fragmented strengths to deduce the spin-orbit splitting.

Recently, we have measured the analyzing powers for the $(\vec{p}, p N)$ reactions from unstable oxygen isotopes ${ }^{14,22-24} \mathrm{O}$ at RIKEN RI Beam Factory using the solid spin-polarized proton target and SHARAQ spectrometer. Details and present status of this study are reported in other articles. ${ }^{16,17}$ 


\section{Studies of Unstable Nuclei at Low Energy}

\subsection{Possible experiments}

One of the future directions of the study of unstable nuclei with the polarized target would be the application to RI-beam experiments at low energies. For example, nucleon transfer reaction at a few tens of $\mathrm{MeV} / \mathrm{A}$ is a well established probe for the spectroscopy of single particle/hole states. A number of experiments have been carried out using spin-polarized light-ion beams and stable targets, where $(\vec{d}, p)$ and $(\vec{p}, d)$ reactions have been measured. Another promising method is the proton resonant scattering at several $\mathrm{MeV} / \mathrm{A}$, where single-particle like resonances can be observed with a good energy resolution of several tens of $\mathrm{keV}$ and with relatively large cross sections of several hundred mb. Another advantage is that particle unbound states are also accessible.

In both reactions above, vector analyzing powers provide us clear assignment of the total angular momentum of the states. Here, we focus on the proton resonant scattering, which would be experimentally less difficult. Proton resonant scattering from unstable nuclei can be measured with the thick target method in inverse kinematics (TTIK). ${ }^{18}$ Excitation function can be scanned with a single incident energy using this method. Combined analysis of the excitation function and vector analyzing power will enable model independent assignment of the spin parity of the resonances, ${ }^{19}$ and also would be sensitive to the configuration mixing. In addition, this method would be effective in resolving extremely wide resonances overlapping with each others, which are often seen in the nuclei near and above the drip line.

Another possibility of the target application would be to polarize the RIs embedded in the target by transferring electron or proton-spin polarization with the cross polarization method. This would allow us to measure the magnetic moment or the RIs by the beta-NMR method.

\subsection{Development of target for low-energy RI-beam experiments}

In order to apply the current solid spin-polarized proton target system to low-energy beam experiments, several modifications are required. First, the material thickness of the whole system must be reduced. The target thickness will be reduced to $100 \mu \mathrm{m}$ $\left(11 \mathrm{mg} / \mathrm{cm}^{2}\right)$. The cooling gas needs be removed. Second, the target material must be changed because the naphthalene rapidly sublimates in vacuum. We adopted $p$ terphenyl which is stable in vacuum and is known to be relatively highly-polarizable even at room temperature.

The challenge is therefore to obtain a high proton-spin polarization in a thin $p$-terphenyl crystal at room temperature. It has been tested at Osaka Univ., where proton polarization of $12.9 \%$ was obtained under magnetic field of $0.4 \mathrm{~T}$ in a $p$ terphenyl crystal with a thickness and an area of $7 \mu \mathrm{m}$ and $3 \mathrm{~mm}^{2}$, respectively. ${ }^{20}$ Fabrication of thin and large single crystal is being tested at RIKEN. A key element required for improving the proton polarization is a light source for optical 
excitation. An LD-pumped fiber laser with a wavelength of $589 \mathrm{~nm}$, which matches to the deepest absorption peak of pentacene molecule, has been newly developed at RIKEN. ${ }^{21}$ High proton polarization of $41 \%$ was obtained under $0.6 \mathrm{~T}$ and at room temperature. ${ }^{22}$ In addition, we have recently tried transferring proton-spin polarization to ${ }^{13} \mathrm{C}$ nuclei under $0.2 \mathrm{~T}$ and at room temperature. High proton-spin polarization at room temperature enables us to produce ${ }^{13} \mathrm{C}$ polarization with an enhancement of three thousand, ${ }^{23}$ which serves as a fundamental development for polarizing radioactive isotopes stopped in the target.

\section{Acknowledgments}

This work was supported by JSPS KAKENHI Grant Numbers 25800155, 23654141 and 25887054 and 18-11398, and by RIKEN President's discretionary fund.

\section{References}

1. J. Dobaczewski et al., Phys. Rev. Lett. 72, 981 (1994).

2. M. M. Sharma, G. A. Lalazissis et. al., Phys. Rev. Lett. 72, 1431 (1994).

3. B. S. Pudliner et al., Phys. Rev. Lett. 76, 2416 (1996).

4. T. Otsuka et al., Phys. Rev. Lett. 95, 232502 (2005).

5. T. Wakui, in Proc. of XIth Int. Workshop on Polarized Ion Sources and Polarized Gas Targets 2005, World Scientific, Singapore (2007).

6. S. Goertz,W. Meyer, and G. Reicherz, Prog. in Part. and Nucl. Phys. 49, 403 (2002).

7. A. Henstra, P. Dirksen, and W. T. Wenckebach, Phys. Lett. A 134, 134 (1988).

8. D. J. Sloop et al., Jour. Chem. Phys. 75, 3746 (1981).

9. H. van Kesteren, W. Wenckebach, and J. Schmidt, Phys. Rev. Lett. 55, 1642 (1985).

10. M. Iinuma, Doctoral Dissertation, Kyoto University (1997).

11. T. R. Eichhorn et al., Nucl. Instr. and Meth. A 754, 10 (2014).

12. T. Uesaka, S. Sakaguchi et al., Phys. Rev. C 82, 021602(R) (2010).

13. S. Sakaguchi, Y. Iseri et al., Phys. Rev. C 84, 024604 (2011).

14. S. Sakaguchi, T. Uesaka et al., Phys. Rev. C 87, 021601(R) (2013).

15. G. Jacob et al., Phys. Lett. 45B, 181 (1973).; P. Kitching et al., Phys. Rev. Lett. 37, 1600 (1976).

16. S. Kawase, T.L. Tang, T. Uesaka et al., in Proc. of The 2nd Conference on Advances in Radioactive Isotope Science" (ARIS2014), to be published.

17. T. L. Tang et al., RIKEN Accelerator Progress Report 2014, to be published.

18. K.P. Artemov et al., Sov. J. Nucl. Phys. 52, 408 (1990).

19. T. Teranishi et al., AIP Conf. Proc. 1525, 552 (2013).

20. K. Tateishi et al., J. Phys. Soc. Jpn. 82, 084005 (2013)

21. S. Wada et al. at RIKEN Optical Green Technology Unit, private communication.

22. K. Tateishi and T. Uesaka et al., in preparation.

23. T. Kawahara, Doctoral Dissertation, Toho University (2015). 